\title{
Research Paper: Effect of Custom-Molded Insole With New Technique on Pain and Function in Females With Flexible Flat Foot: A Pilot Study
}

\author{
Fatemeh Bahramian ${ }^{1}$, Atefeh Abootorabi ${ }^{1}$, *atemeh Zarezadeh ${ }^{1}$, Tahmine Rezaeian ${ }^{2}$
}

1. Department of Orthotics \& Prosthetics, University of Social Welfare and Rehabilitation Sciences, Tehran, Iran. 2. Department of Biomedical Engineering, Faculty of Biological Sciences, University of Leeds, Leeds, England.

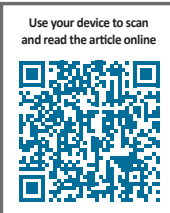

Citation: Bahramian F, Abootorabi A, Zarezadeh F, Rezaeian T. [Effect of Custom-Molded Insole With New Technique on Pain and Function in Females With Flexible Flat Foot: A Pilot Study (Persian)]. Journal of Rehabilitation. 2017; 17(4):318-325. http://dx.doi.org/ 10.21859/jrehab-1704318

doi): http://dx.doi.org/ 10.21859/jrehab-1704318

Received: 5 May 2016 Accepted: 9 Aug. 2016

Keywords:

Flat foot, Insole, Foot orthoses, Medial heel skive, Pain, Function

\section{A B STRACT}

Objective A flat foot is a complex postural deformity in which the medial longitudinal arch is decreased. It is associated with diverse symptoms and varying degrees of deformity and disability. This disorder can sometimes contribute to problems in the ankles and knee as it can alter the alignment. Conservative treatments are used to treat patients with a flat foot. One common treatment is the use of insoles with medial heel skive technique. The purpose of this study was to evaluate the effect of custom-molded insoles with new technique on pain and function in females with a flexible flat foot.

Materials \& Methods This research is a quasi-experimental study of before-and-after type that included 12 subjects with a flexible flat foot from the Social Welfare and Rehabilitation Sciences University of Tehran. To provide a negative cast, plaster cast impressions were taken for each participant's foot. According to the method of modification (medial heel skive) that was introduced by Kirby, the positive cast of the foot was corrected. Each person received an insole with medial heel skive technique. The pain and function of each foot were evaluated with a visual analogue scale and Foot and Ankle Outcome Score before applying the treatment and at six weeks follow-up. Paired t-test and non-parametric tests were used for statistical analysis.

Results It was found that the pain and function significantly improved after 6 weeks of using insoles with medial heel skive technique in subjects with flat foot $(P<0.001)$.

Conclusion According to our findings, there was a significant difference in pain and function in patients with a flat foot through medial heel skive technique. Therefore, it seems that the insoles can be an effective prescription for subjects with a flat foot.

\section{* Corresponding Author:}

Address: Department of Orthotics \& Prosthetics, University of Social Welfare and Rehabilitation Sciences, Koodakyar Ave, Daneshjoo Blvd, Evin, Tehran, Iran.

Tel: +98 (21) 22180010

E-Mail: natel_ortho@yahoo.com 


\title{
بررسى ثأثير كفى سفارشى ساختهشده با شيوه جديد بر ميزان درد و عملكرد در زنان مبتلا به صافى كف يا انعطاف يذير: مطالعه مقدماتي
}

فاطمه بهراميان'، عاطفه ابوترابى'، "فاطمه زارعزاده'، تهمينه رضائيان'

ا - كروه ارتويدى فنى، دانشعاه علوم بجزيستى و توانبخشى، تهران، ايران.

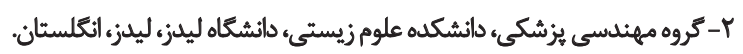

\begin{abstract}
حكSe

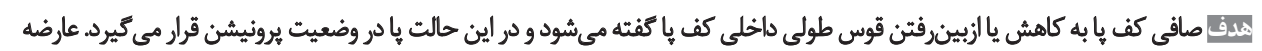

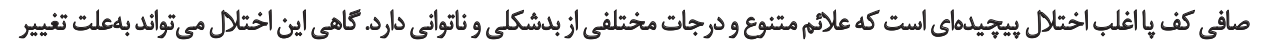

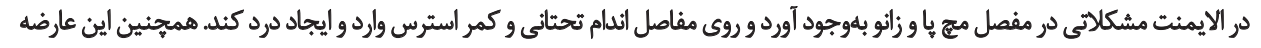

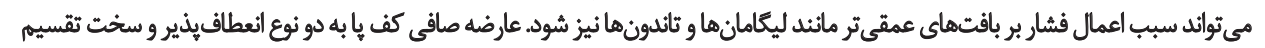

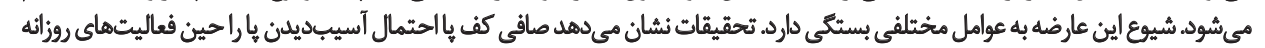

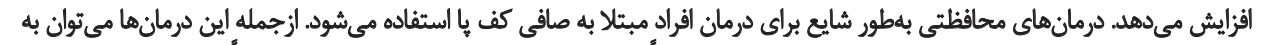

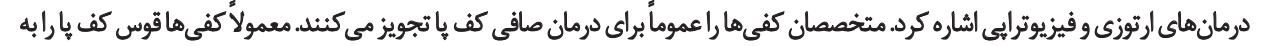

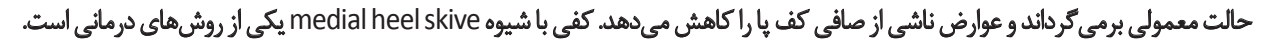

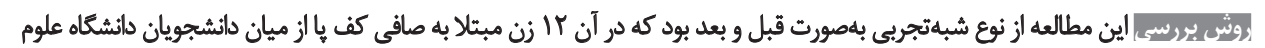

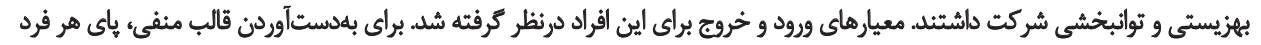

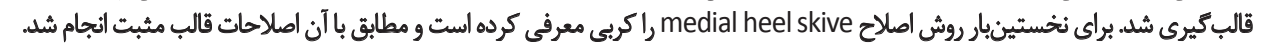

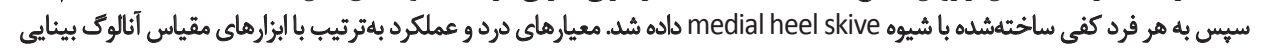

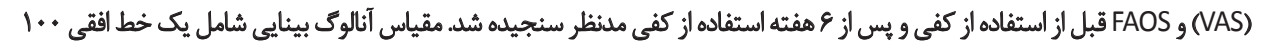

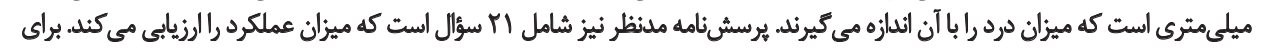

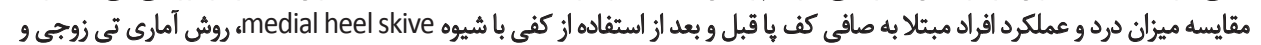

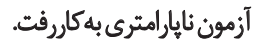

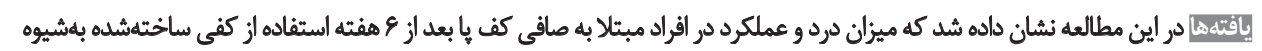
medial heel skive

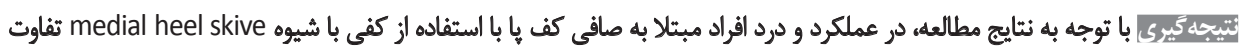

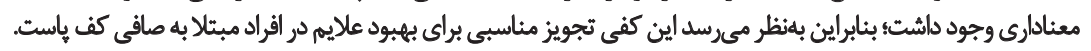

تاريخ دريافت: 194

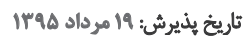

كليدوازٔهها:

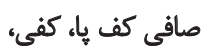

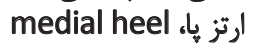
skive

آن به نوعى اطلاق مى شود كه طى تحمل وزن قوس كف ئ ياصاف

مقدمه

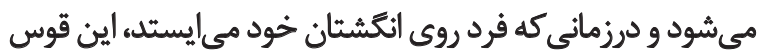

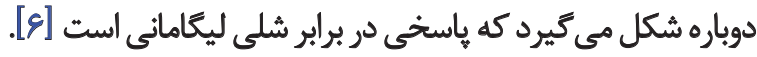

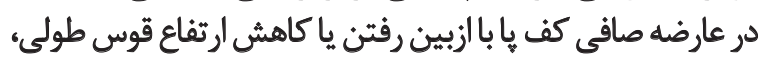

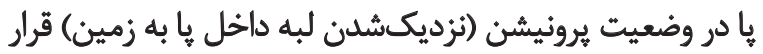

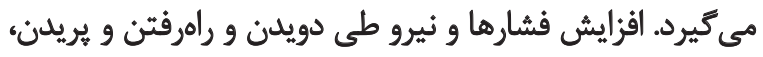

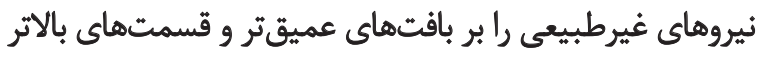

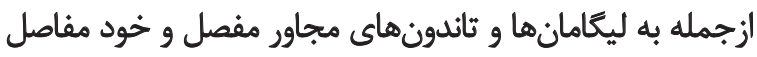

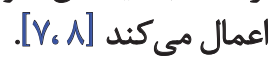

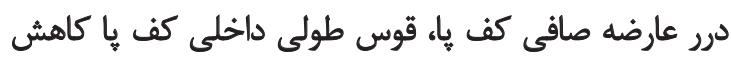

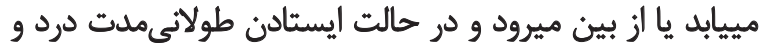

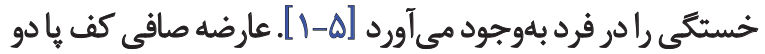

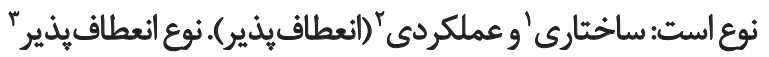

1. Structural flat foot

2. Functional flat foot

3. Flexible flat foot

: ثاطمهزينده مسئول:

فاطمه زارعزانه

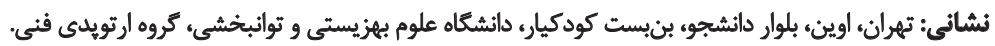

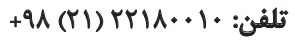

رايانامه: natel_ortho@yahoo.com 


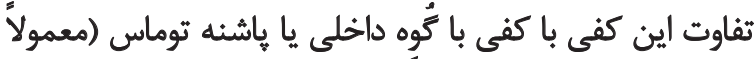

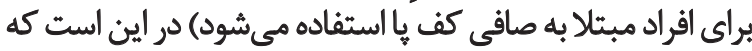

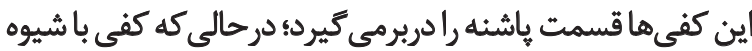
medial heel skive

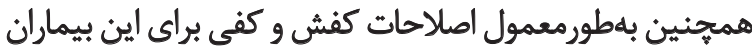

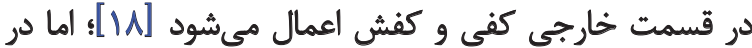

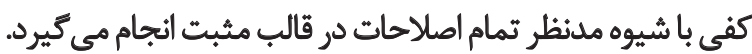

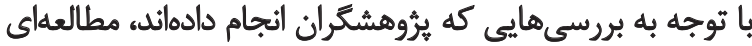

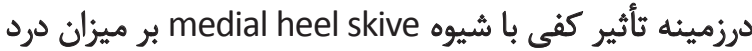

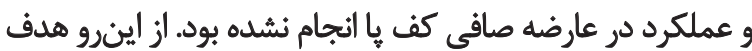

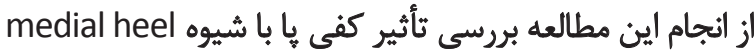

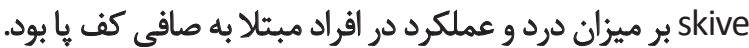

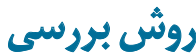

در اين مطالعه شبهتجربى، ب ا زن مبتلابه صافى كف يَاشركت

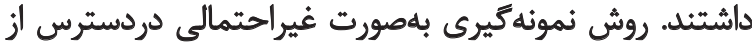

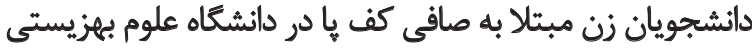

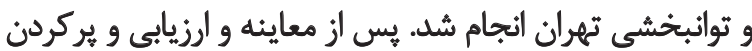

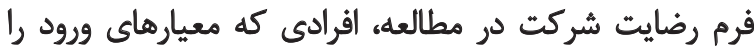

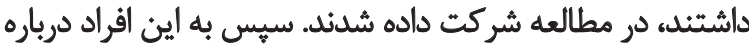

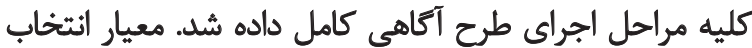

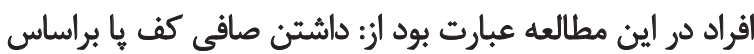

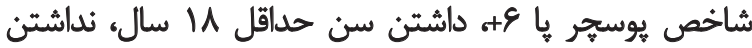

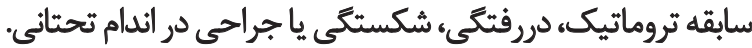

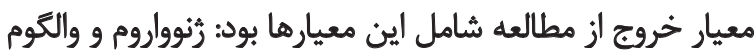

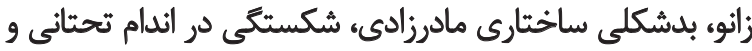

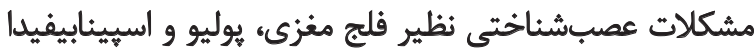

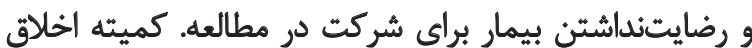

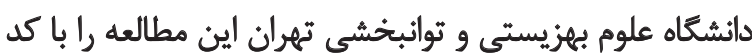

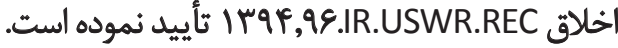

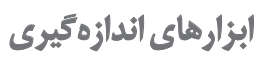

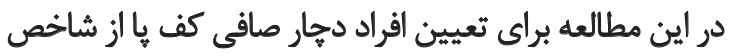

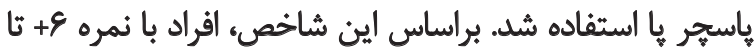

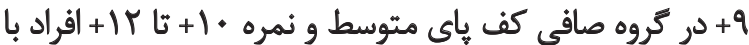

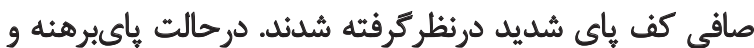

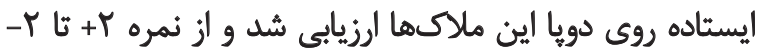

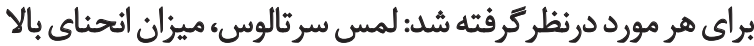

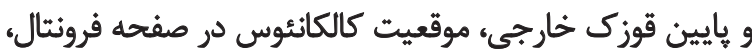

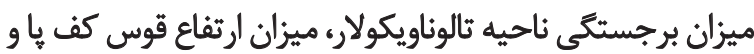

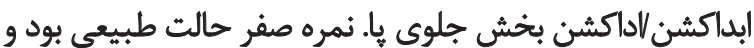

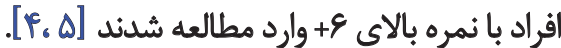
براى ارزيابى ميزان بهبود درد و شدت درد ادراكشده از ابزار

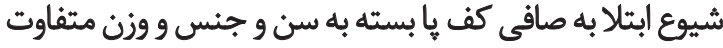

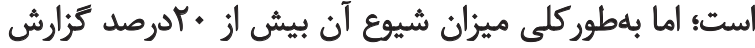

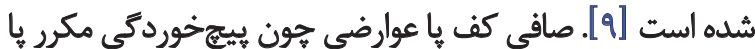

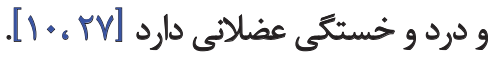

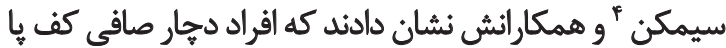

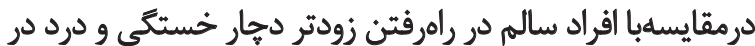

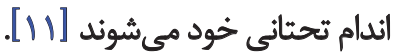

كوفمن ه (999 (19) در مطالعه خود به اين نتيجه رسيد كه صافي

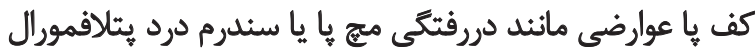

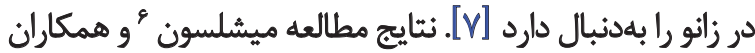

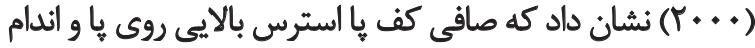

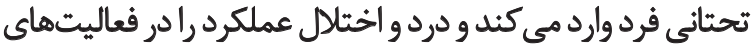

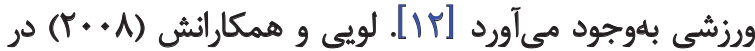

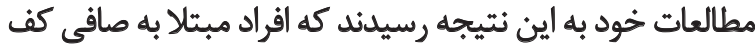

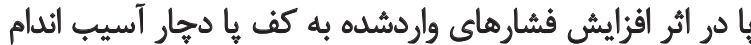

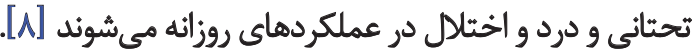

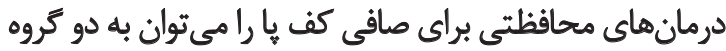

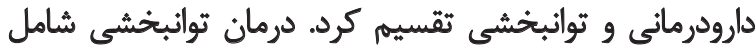

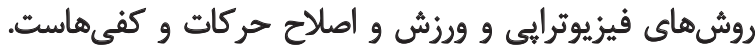

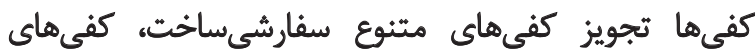

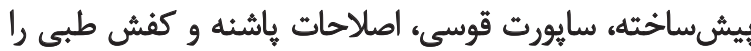

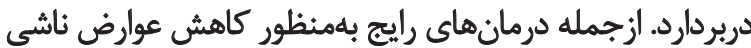

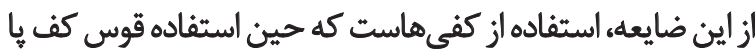

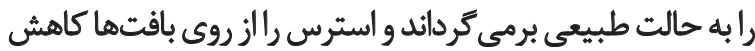

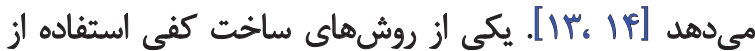

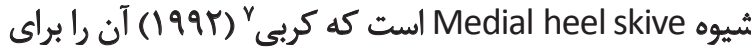

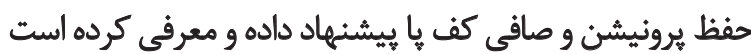

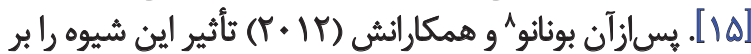

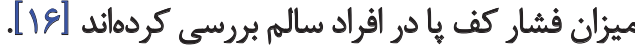

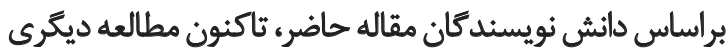

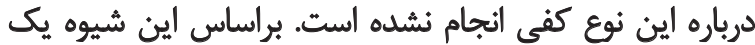

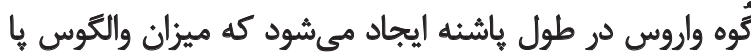

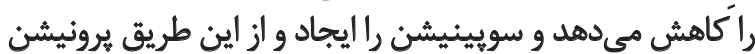

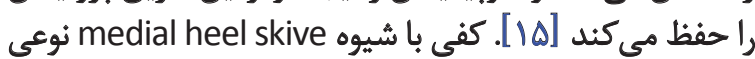

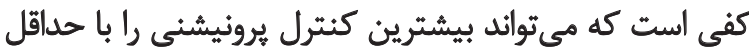

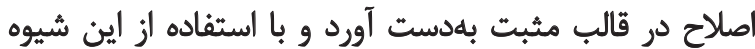

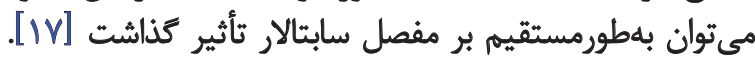

4. Simkin

5. Kaufman

6. Michelson

7. Kirby

8. Bonanno 


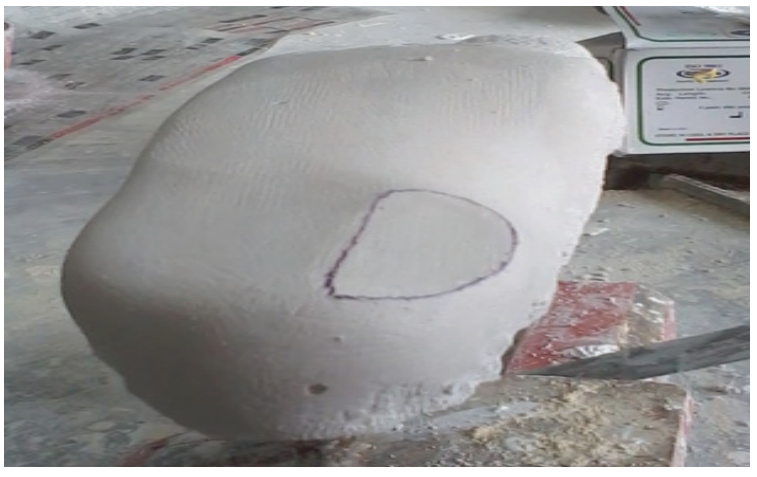

توانبخننى

تصوير ا. اصلاح كفي با شيوه medial heel skive.

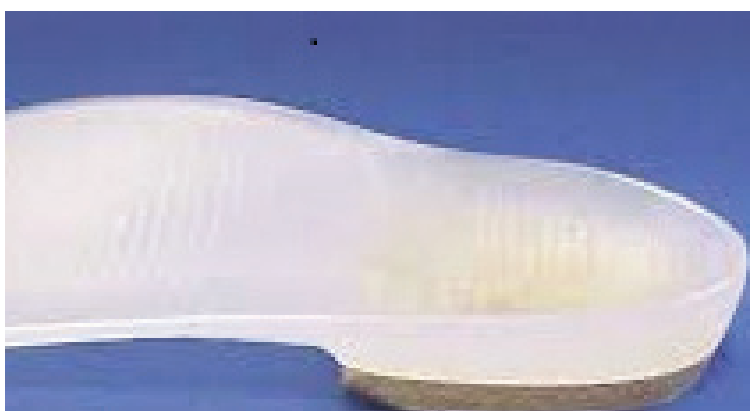

توانبفننى

تصوير Y. كفى با شيوه medial heel skive.

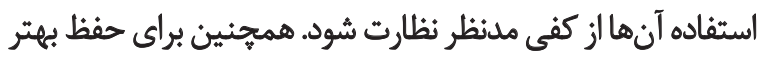
شرايط مطالعه، افراد از كفشهائ يكسان استفاده كردند.

تحليل آمارى دادهها

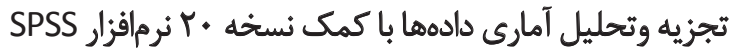

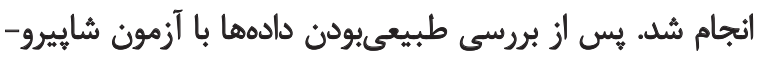

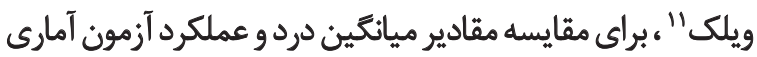

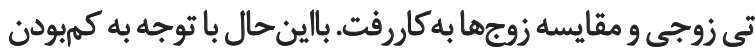

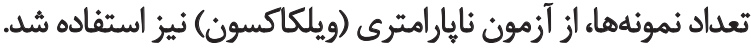

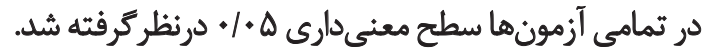

يافتهها

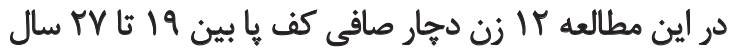

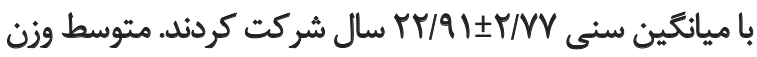

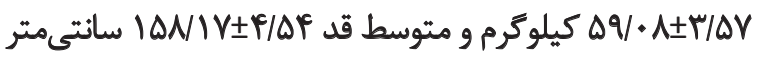

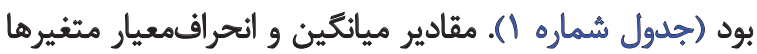

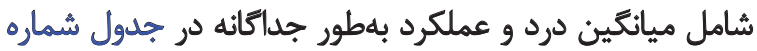

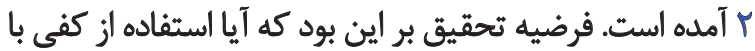

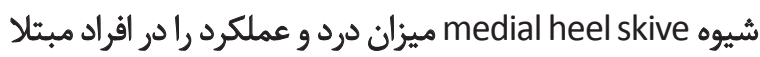
به صافى كف با بهبود مىبخشد يا خير. نتايج تحقيق نشان دردان داد ماد
مقياس آنولوك بينايى (VAS)" استفاده شد. اين مقياس شامل

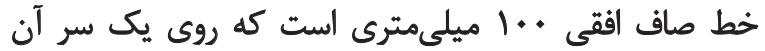

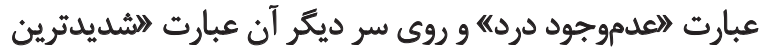

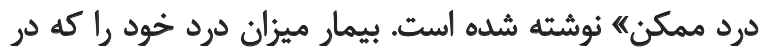

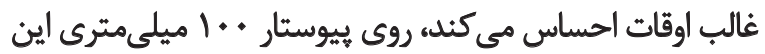

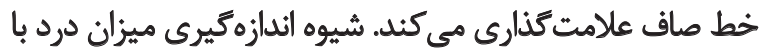

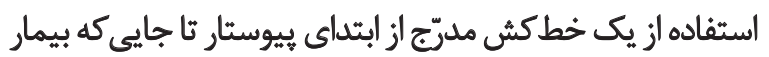

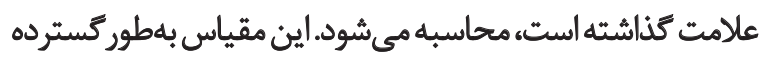
در يروهشهاى مرتبط با درد استفاده و اعتبار و واياييى آن بارها

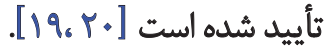

براى ارزيابى عملكرد اين افراد، از بخش عملكرد فعاليتهاى

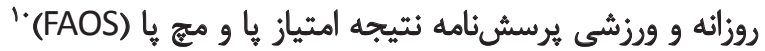

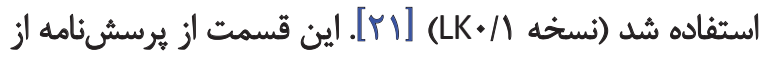

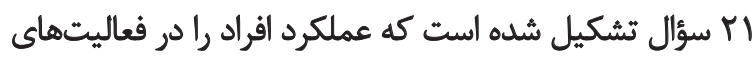

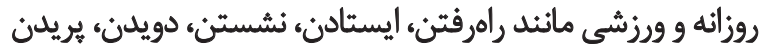

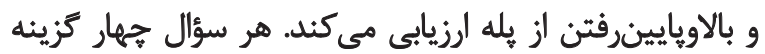

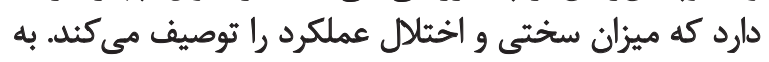

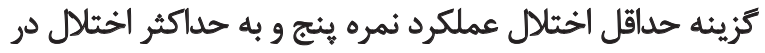

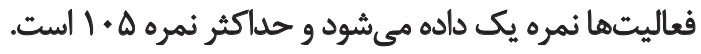
براى ساخت كفى از ياى هر فرد بلهصورت جدامانه قالبكيرى

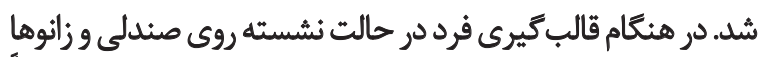

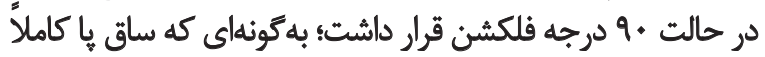

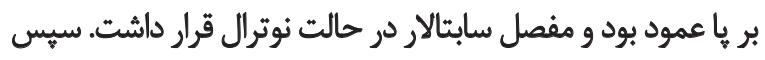

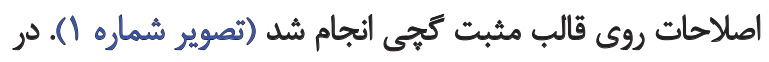

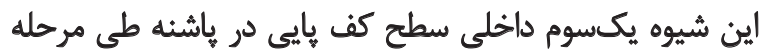

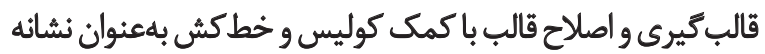

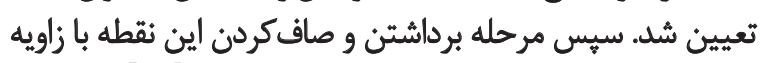

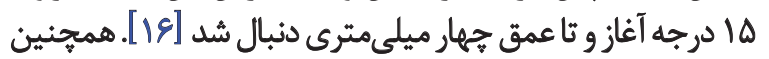

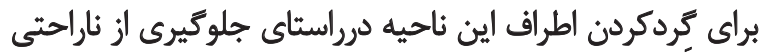

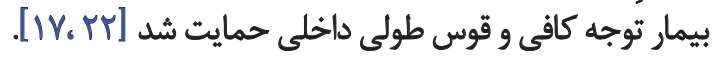

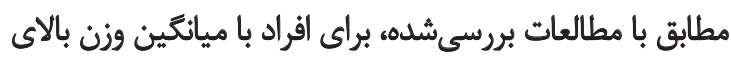

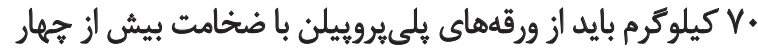

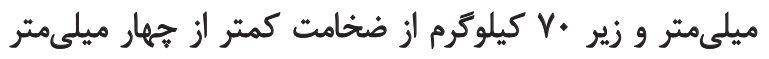

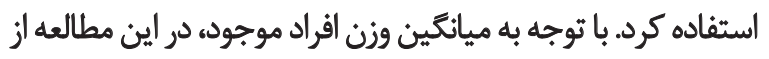

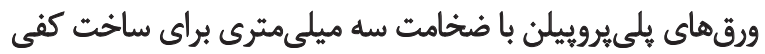

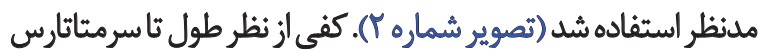

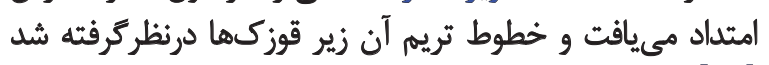

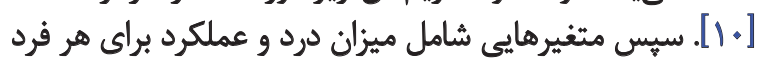

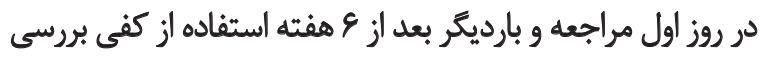

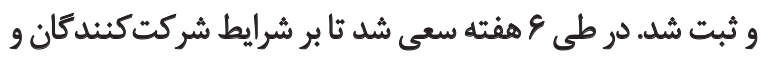


جدول ا. ميانكين و انحراف استاندارد نمودار آزمونئىها در اطلاعات شخصي.

\begin{tabular}{|c|c|c|c|c|}
\hline كمترين مقدار & بيشترين مقدار & انحرافمعيار & مياتكين & متغيرها \\
\hline 19 & r & $T / W$ & $r r / q)$ & سن (سال) \\
\hline$\Delta F$ & so & $r / \Delta Y$ & $\Delta Q \cdot 1$ & وزن (كيلوكرم) \\
\hline laP & iv. & $\varphi(\Delta)$ & IV/NQA & قد (سائتى مثر) \\
\hline+8 & $+\gamma$ & $r / q q Y$ & $9 / \pi$ & شاخص باسجر ها \\
\hline
\end{tabular}

توانبخننى

جدول I. بروسي توزيع طبيعي متغيرها شاييروو ويلك (Shapiro-Wilk).

\begin{tabular}{|c|c|c|c|}
\hline مقدار احتمال & انحرافمعيار & مياتكين & متغيرها \\
\hline - $M N$ & $1 / 10$ & $F / \pi$ & درد \\
\hline - NAS & $\Delta / \Delta F$ & SNTA & عملكرد \\
\hline
\end{tabular}

توانبخننى

جدول "ا. ميانكين و انحراف استاندارد نمرات درد و عملكرد در آزمونىىها.

\begin{tabular}{|c|c|c|c|c|c|c|c|}
\hline \multicolumn{2}{|c|}{ آزمون ناهارامترى } & \multicolumn{3}{|c|}{ بعد از استفاده از كفى } & \multicolumn{2}{|c|}{ قبل از استفاده از كفى } & \multirow[t]{2}{*}{ متغيرها } \\
\hline مقدار احتمال & اختاف مياتكينها & مقدار احتمال & انحرافسمعيار & مياتكين & اتحرافمعيار & مياتكين & \\
\hline .1 .4 & T/TA & $<\cdot 1.0)^{*}$ & . NaY & $Y / \bullet \Lambda$ & $1 / 10$ & $F / T$ & درد \\
\hline .1 .08 & reigr & $<\odot / .\left.\bullet\right|^{*}$ & A/TE & १४/৭Y & $\Delta / \Delta F$ & ENTA & عملكرد \\
\hline
\end{tabular}

توانبخننى

صافي كف پا راطي تحمل وزن، راهرفتن، بالاويايينرفتن از يلكان،

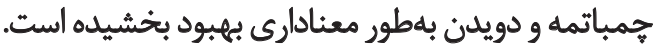

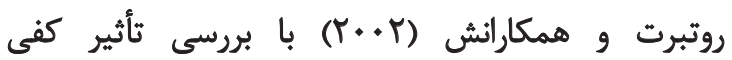

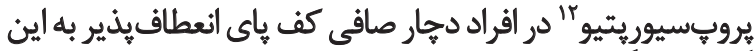

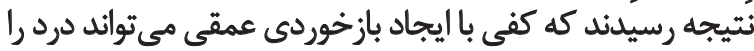

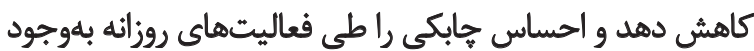

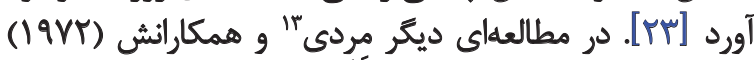

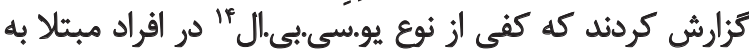

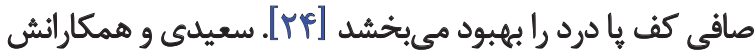

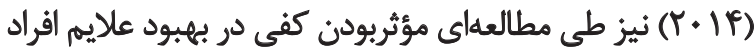

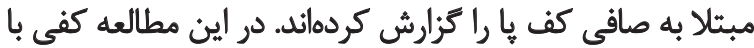

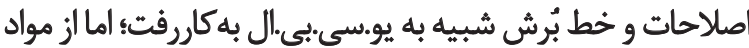

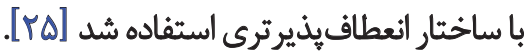

نتايج مطالعه مرورى كالينز و همكارانش (V) • • (Y) نشاندهنده

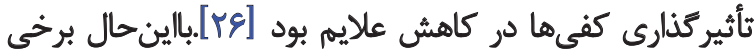

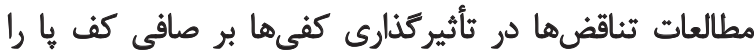

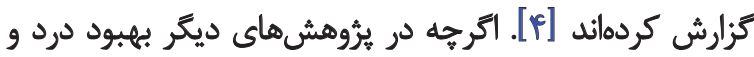

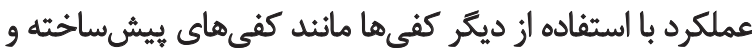

\section{Proprioceptive}

13. Mereday

14. UCBL
كه كفى با شيوه medial heel skive سبب كاهش معنى دار درد

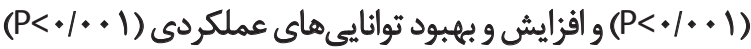

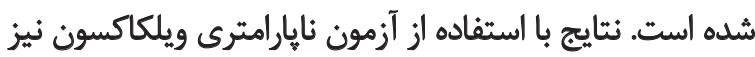
تأييد شده است (جدول شماره شب).

ث

هدف از انجام اين مطالعه بررسى تأثير كفى سفارشى ساختهشده

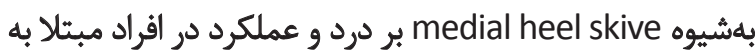
صافى كف يا بود. در اين مطالعه نشان داده شد كه كفي با با شيوه medial heel skive

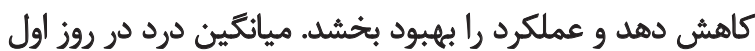

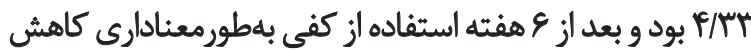

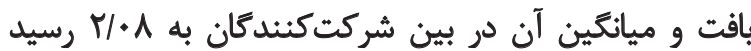

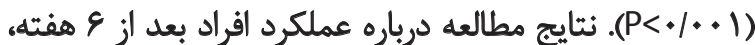

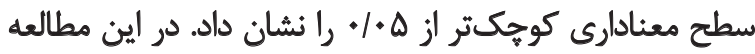

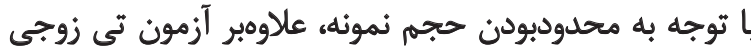

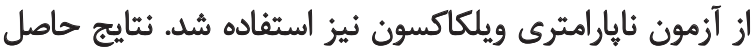

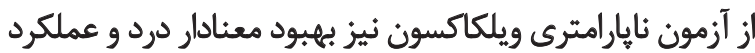

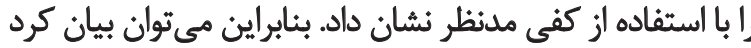

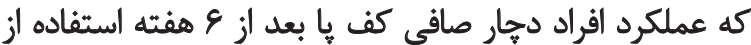

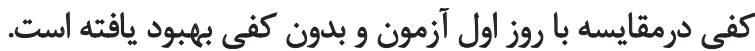

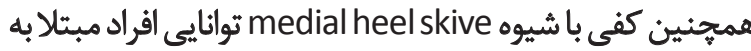




\section{References}

[1] Ledoux WR, Hillstrom HJ. The distributed plantar vertical force of neutrally aligned and pes planus feet. Gait \& Posture. 2002; 15(1):1-9. doi: 10.1016/s0966-6362(01)00165-5

[2] Queen RM, Mall NA, Nunley JA, Chuckpaiwong B. Differences in plantar loading between flat and normal feet during different athletic tasks. Gait \& Posture. 2009; 29(4):582-86. doi: 10.1016/j. gaitpost.2008.12.010

[3] Evans AM, Rome K. A review of the evidence for non-surgical interventions for flexible pediatric flat feet. European Journal of Physical and Rehabilitation Medicine. 2011; 47(1):69-89.

[4] Dehghani F, Saeedi H. [Investigating the Influence of prefabricated insole with medial flange on forefoot and rearfoot alignment changes at females with flexible flat foot (Persian)]. Journal of Rehabilitation. 2015; 15(4):70-77.

[5] Aboutorabi AT, Zarezadeh F, Saeedi H, Maleki M, Fardipour S. [Effect of orthopedic shoe and functional foot orthosis on static equilibrium in flexible flat foot juvenile (Persian)]. Journal of Rehabilitation. 2014; 15(3):10-17.

[6] Kuhn DR, Shibley NJ, Austin WM, Yochum TR. Radiographic evaluation of weight-bearing orthotics and their effect on flexible pes planus. Journal of Manipulative \& Physiological Therapeutics. 1999; 22(4):221-6. doi: 10.1016/s0161-4754(99)70048-5

[7] Kaufman KR, Brodine SK, Shaffer RA, Johnson CW, Cullison TR. The effect of foot structure and range of motion on musculoskeletal overuse injuries. The American Journal of Sports Medicine. 1999; 27(5):585-93.

[8] Levy JC, Mizel MS, Wilson LS, Fox W, McHale K, Taylor DC, et al. Incidence of foot and ankle injuries in West Point cadets with pes planus compared to the general cadet population. Foot \& Ankle International. 2006; 27(12):1060-4.

[9] Bouchard M, Mosca VS. Flatfoot deformity in children and adolescents: surgical indications and management. Journal of the American Academy of Orthopaedic Surgeons. 2014; 22(10):62332. doi: 10.5435/jaaos-22-10-623

[10] Tachdjian M. Disorders of the foot. In: Ricco AI, Richards BS, Herring JA, editors. Tachdjian's Pediatric Orthopaedics. $5^{\text {th }}$ ed. Philadelphia: Saunders; 1990, p. 761-833.

[11] Simkin A, Leichter I, Giladi M, Stein M, Milgrom C. Combined effect of foot arch structure and an orthotic device on stress fractures. Foot \& Ankle. 1989; 10(1):25-9. doi: $10.1177 / 107110078901000105$

[12] Michelson J, Durant D, McFarland E. The injury risk associated with pes planus in athletes. Foot \& Ankle International. 2002; 23(7):629-33. doi: 10.1177/107110070202300708

[13] Tsung BYS, Zhang M, Mak AF, Wong MWN. Effectiveness of insoles on plantar pressure redistribution. Journal of Rehabilitation Research and Development. 2004; 41(6):767. doi: 10.1682/ jrrd.2003.09.0139

[14] Paiehdar S, Saeedi H, Ahmadi A, Kamali M, Mohammadi M. [The comparison of the immediate effect of 3 functional, UCBL and modified UCBL foot orthotics impact on dynamic balance in
كفي هاي داراى حس عمقي نيز نشان داده شيده است؛ اما شيوه

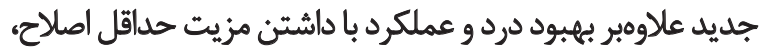

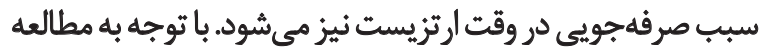

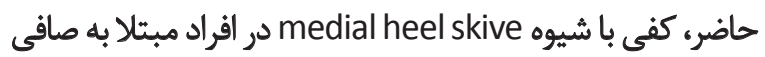

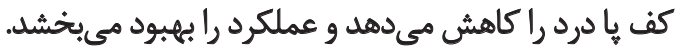

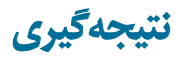
با درنظركرفتن هدف اين مطالعه كه بروسى ثأثير كفى

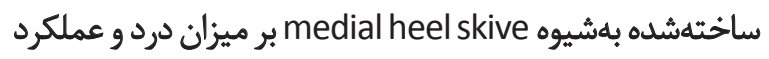

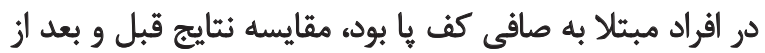

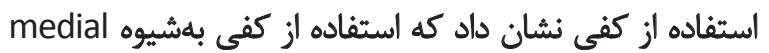
heel skive

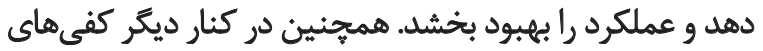

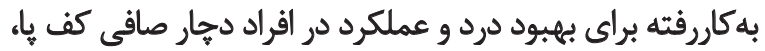

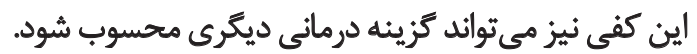

loctsolo

مههمترين محدوديتهاي مطالعه حاضر عبارت بود از: كمبودن

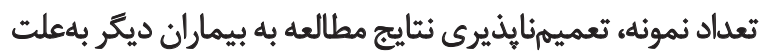

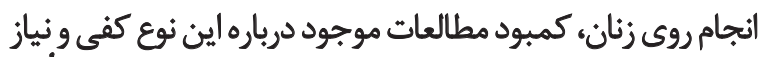

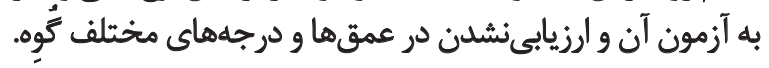

يشئهاsها

براساس هافتههاي يُروهش حاضر بيشينهاد مي شود تحقيق

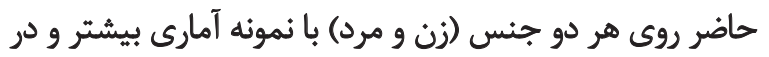
عمق هاي مختلف صورت كيرد. همجينين در مطالعات آينده كفي

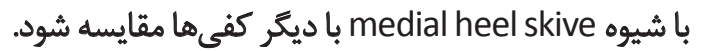

$$
\text { تشيكر وقدرداني }
$$

اين مقاله فاقد حامى مالى بوده و دريايان، يُروهشكَران از تمامى توانى

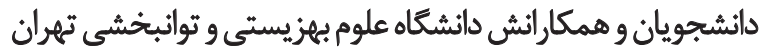

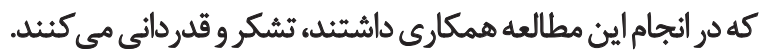


subjects with flexible flatfoot (Persian)]. Journal of Rehabilitation. 2014; 14(4):66-73.

[15] Kirby KA. The medial heel skive technique. Improving pronation control in foot orthoses. Journal of the American Podiatric Medical Association. 1992; 82(4):177-88. doi: 10.7547/8750731582-4-177

[16] Bonanno DR, Zhang CY, Farrugia RC, Bull MG, Raspovic AM, Bird AR, et al. The effect of different depths of medial heel skive on plantar pressures. Journal of Foot and Ankle Research. 2012; 5(1):9. doi: 10.1186/1757-1146-5-s1-o9

[17] Kirby KA. Evolution of foot orthoses in sports. Athletic footwear and orthoses in sports medicine. Philadelphia: Springer; 2010

[18] Zamosky I. Shoe modifications in lower-extremity orthotics. Journal of Rehabilitation Research and Development. 2013; 50(6). doi: 10.1682/JRRD.2013.02.0046

[19] Price DD, McGrath PA, Rafii A, Buckingham B. The validation of visual analogue scales as ratio scale measures for chronic and experimental pain. Pain. 1983; 17(1):45-56. doi: 10.1016/03043959(83)90126-4

[20] Turk DC, Robinson JP. Assessment of patients with chronic pain: a comprehensive approach. In: Turk DC, Melzack R, editors. Handbook of Pain Assessment. $3^{\text {rd }}$ ed. New York, NY: Guilford Press; 2011, p. 188-210

[21] Mani SB, Brown HC, Nair P, Chen L, Do HT, Lyman S, et al Validation of the foot and ankle outcome score in adult acquired flatfoot deformity. Foot \& Ankle International. 2013; 34(8):114046. doi: $10.1177 / 1071100713483117$

[22] Kirby KA. Subtalar joint axis location and rotational equilibrium theory of foot function. Journal of the American Podiatric Medical Association. 2001; 91(9):465-87. doi: 10.7547/87507315-91-9-465

[23] Rothbart BA. Medial column foot systems: an innovative tool for improving posture. Journal of Bodywork \& Movement Therapies. 2002; 6(1):37-46. doi: 10.1054/jbmt.2001.0261

[24] Mereday C, Dolan CM, Lusskin R. Evaluation of the University of California Biomechanics Laboratory shoe insert in "flexible" pes planus. Clinical Orthopaedics \& Related Research. 1972; 82:45-58.

[25] Saeedi H, Mousavi ME, Majddoleslam B, Rahgozar M, Aminian G, Tabatabai Ghomshe F, et al. The evaluation of modified foot orthosis on muscle activity and kinetic in a subject with flexible flat foot : single case study. Prosthetics \& Orthotics International. 2014; 38(2):160-6. doi: 10.1177/0309364613492170

[26] Collins N, Bisset L, McPoil T, Vicenzino B. Foot orthoses in lower limb overuse conditions: a systematic review and meta-analysis. Foot \& Ankle International. 2007; 28(3):396-412. doi: 10.3113/ fai.2007.0396

[27] Askary Kachoosangy R, Aliabadi F, Ghorbani M. Prevalence of flat foot: comparison between male and female primary school students. Iranian Rehabilitation Journal. 2013; 11(2):22-24. 
